\title{
Contribuições da antropologia para uma abordagem das questões relativas à saúde do idoso
}

\author{
An anthropological approach to senior \\ citizens' health issues
}

Elizabeth Uchôa ${ }^{1}$

\footnotetext{
1 Núcleo de Estudos em Saúde Pública e Envelhecimento, Centro de Pesquisas René Rachou, Fundação Oswaldo Cruzl Universidade Federal de Minas Gerais

Av. Augusto de Lima, 1715, Belo Horizonte, $M G$ 30190-002, Brasil. uchoae@cpqrr.fiocruz.br
}

\begin{abstract}
This paper discusses the specificity of the anthropological approach to aging. The theoretical framework is introduced and illustrated by presenting studies carried out in different societies. These studies describe a great diversity of forms of aging and experiences relative to growing old that allow us to distinguish two kinds of elements that influence aging: those inherent to aging as a biological process and those directly associated with the characteristics of the individual, the social dynamics, and prevailing public policies in each society. The anthropological approach considers the interaction between these two groups of factors.
\end{abstract}

Key words Anthropology; Aging; Aging Health

Resumo Discute-se a especificidade da abordagem antropológica para a investigação de questões relativas à saúde do idoso. Alguns estudos sobre o envelhecimento realizados em diferentes culturas são utilizados como exemplos da variedade de formas de envelhecer e de experiências relativas ao envelhecimento, levando à distinção de dois tipos de elementos que influenciam este processo: aqueles intrínsecos ao envelhecimento biológico e os relacionados às características do indivíduo, à dinâmica social e às políticas públicas vigentes na sociedade em questão. Uma abordagem antropológica leva em conta a articulação entre estes dois tipos de elementos e o papel específico de cada um deles na construção de maneiras típicas de envelhecer, na identificação de problemas de saúde e no desenvolvimento de estratégias para enfrentá-los. Ressalta-se a potencialidade da abordagem antropológica no campo da Saúde Pública contemporânea.

Palavras-chave Antropologia; Envelhecimento; Saúde do Idoso 


\section{Introdução}

O envelhecimento da população brasileira vem ocorrendo muito rapidamente, verificando-se um importante aumento da população idosa, tanto em termos absolutos quanto proporcionais (Berquó, 1999). Fazendo eco ao panorama mundial, este fato revela indicadores positivos de uma melhora na qualidade de vida da população, mas faz surgir novas demandas para os serviços de saúde, gera aumentos substanciais nos custos de programas médicos e sociais e cria um novo desafio: assegurar à crescente população de idosos o atendimento médico que lhe é de direito (Birren, 1983; Ducharme, 1992; Fournier, 1989; Neugarten, 1967; Veras, 1992). Assim, no início deste novo milênio, o acelerado crescimento da população idosa brasileira surge como um elemento central para a elaboração de novas políticas públicas.

Neste contexto, trata-se de identificar quais são os problemas prioritários para a população idosa brasileira e de definir que ações devem ser privilegiadas para enfrentar esses problemas. Estudos epidemiológicos são essenciais para identificar problemas prioritários, de modo a orientar decisões relativas à definição de prioridades para intervenção. Entretanto, quando o foco de interesse desloca-se da definição de problemas prioritários em saúde, para as ações que devem ser privilegiadas para resolvê-los, os estudos antropológicos tornam-se imprescindíveis. Para que possamos desenvolver intervenções adequadas às características sociais e culturais da população idosa, é preciso conhecer um pouco mais sobre a maneira como os idosos brasileiros envelhecem, como atribuem significado a este período de suas vidas ou como o integram à sua experiência. De maneira particular, é preciso conhecer um pouco mais sobre a forma como o idoso percebe seus problemas de saúde, como procura resolvê-los e quais são as dificuldades que encontra nesse percurso.

Discutem-se, aqui, a especificidade e a potencialidade da abordagem antropológica para o estudo das questões relativas à saúde do idoso. Elementos conceituais serão introduzidos e exemplificados com a apresentação de estudos realizados em diferentes culturas.

\section{Contribuições da antropologia}

Apesar da entrada tardia no campo de estudos sobre o envelhecimento, a antropologia já deu algumas contribuições fundamentais para inovar a abordagem das questões relativas à saúde do idoso. A primeira delas refere-se à relativização da visão universalista, usualmente adotada em estudos sobre o envelhecimento. Delimitado inicialmente a partir de sua dimensão biológica, o envelhecimento foi associado à deterioração do corpo e, em conseqüência, tratado como uma etapa da vida caracterizada pelo declínio (Corin, 1985; Debert, 1999). Cristalizou-se, assim, uma visão orgânica do envelhecimento. Entretanto, estudos realizados em sociedades não ocidentais tornaram conhecidas imagens bem mais positivas da velhice e do envelhecimento, questionando a universalidade da visão ocidental e ensinando que uma representação de velhice enraizada nas idéias de deterioração e perda não é universal.

Um exemplo clássico desta outra visão da velhice nos foi dado, há mais de cinqüenta anos, por Evans-Pritchard (1989) em seu estudo sobre os Nuer, grupo étnico do Sudão. Segundo este autor, a entrada na adolescência para o Nuer do sexo masculino é marcada por um ritual de iniciação que define sua inserção em uma classe de idade e determina seu estatuto de superioridade, igualdade ou inferioridade frente aos outros Nuer. Os membros de uma classe de idade devem respeito aos da classe anterior, que é composta por pessoas mais velhas e, portanto, superiores na hierarquia social. Estes respeito e deferência transparecem em todas as dimensões da vida social.

Um exemplo atual nos é dado pelos $\mathrm{Bam}$ bara do Mali, grupo com a qual tive oportunidade de trabalhar (Uchoa, 1988). Os Bambara consideram a velhice uma conquista. Para eles, o envelhecimento é concebido como um processo de crescimento que ensina, enriquece e enobrece o ser humano. Ser velho significa ter vivido, ter criado filhos e netos, ter acumulado conhecimento e ter conquistado, através destas experiências, um lugar socialmente valorizado. Os Bambara constituem um exemplo atual da situação privilegiada dos idosos em algumas sociedades africanas. Para os Bambara, a idade é um elemento determinante da posição de cada indivíduo na sociedade. Toda a vida social é organizada segundo o princípio da senioridade. Considera-se que os mais velhos estão mais próximos dos ancestrais e, por esta razão, detêm a autoridade. Respeito e submissão marcam o conjunto de atitudes e comportamentos dos mais jovens para com os mais velhos.

Os comportamentos e atitudes dos jovens inuit (esquimós) para com os idosos constituem um paradoxo que espantou europeus e norte americanos quando estes entraram em contato com a cultura deste povo (Guemple, 1989). Por um lado, eles demonstram carinho e 
afeição para com os parentes mais velhos, mas por outro podem abandoná-los no meio de uma estrada ou ajudá-los a cometer suicídio por afogamento ou estrangulamento. Esta aparente crueldade enraíza-se em uma concepção particular de vida, de morte e da própria essência do ser humano, podendo co-existir, sem contradição, com atitudes de interesse e suporte aos mais velhos da comunidade.

Entretanto de uma maneira geral, estudos realizados em sociedades não ocidentais enfatizam o poder, o elevado status e o papel social central atribuído aos idosos nestas sociedades. Alguns estudos ressaltam o impacto negativo do processo de modernização sobre o status dos idosos nessas sociedades (Cowgill \& Holmes, 1972).

É evidente que não se pode ter uma visão idealizada e homogênea das sociedades não ocidentais, mas é preciso reconhecer a contribuição trazida pelos estudos realizados nessas sociedades. À medida que se documentou o processo do envelhecimento em diferentes culturas e que se constatou a diversidade de formas de envelhecer, a velhice e o envelhecimento deixaram de ser encarados como fatos naturais, para serem encarados como fenômenos profundamente influenciados pela cultura.

Uma segunda contribuição da antropologia, bastante ligada à primeira, refere-se à construção de um quadro conceitual e metodológico inovador. Com o aparecimento dos estudos holísticos, o envelhecimento passa a ser estudado através de uma perspectiva êmica. O pesquisador tenta penetrar em uma cultura específica para descobrir como aspectos relativos à velhice e ao envelhecimento são organizados e adquirem significado. O envelhecimento deixa, então, de ser encarado como um estado ao qual os indivíduos se submetem passivamente para ser encarado como um fenômeno biológico, ao qual os indivíduos reagem a partir de suas referências pessoais e culturais (Corin, 1982; Marshall, 1986, 1987). Esta nova tendência coincide com o desenvolvimento da antropologia interpretativa e encontra em Geertz (1973) subsídios para a construção de um novo paradigma para o estudo da relação entre envelhecimento e cultura. Geertz (1973), que se situa na origem desta corrente, define a cultura como sendo um universo de significados que permite aos indivíduos de um grupo interpretar sua experiência e guiar suas ações. Para ele, a cultura é o contexto que torna inteligíveis os diversos acontecimentos e situações da vida, como por exemplo limitações e perdas tão freqüentes nesta fase da vida. Uma abordagem interpretativa do envelhecimento focaliza a interação entre parâmetros culturais, traços indivi- duais e marcadores biológicos na construção de maneiras típicas de envelhecer e viver o envelhecimento (Corin, 1982; Létourneau, 1989).

Meyerhoff \& Simic (1978) publicaram uma coletânea de estudos antropológicos sobre o envelhecimento. Em todos estes estudos, o envelhecimento é abordado como um fenômeno universal que gera problemas comuns, mas que podem ser vividos e resolvidos diferentemente através das culturas. Os autores focalizam aspectos estruturais, culturais e vivenciais do envelhecimento em cinco sociedades distintas e nos convidam a examinar os problemas específicos do envelhecimento e as estratégias adaptativas utilizadas pelos idosos em termos de uma articulação entre as capacidades do indivíduo e os recursos do meio.

Um outro exemplo vem de um estudo realizado por Arcand (1989) junto aos Cuiva, população indígena da Colômbia. Neste estudo, o autor nos leva a interrogar sobre a existência de modelos culturais que seriam determinantes no aparecimento de problemas específicos do envelhecimento em diferentes sociedades. Segundo o autor, os Cuiva negariam todas as formas de envelhecimento, pois, preocupados com os ideais de igualdade e homogeneidade que estruturam a sociedade, evitariam tudo o que poderia provocar uma ruptura social. Segundo Arcand (1989), os Cuiva não constroem uma idade da velhice: depois de sair da infância, o indivíduo integra o grupo de adultos e continua confundido neste grupo até sua morte. Não existe espaço social particular para os velhos, nem atividades das quais eles são excluídos. Entre os Cuiva, ninguém é considerado velho demais para produzir, tomar decisões, casar ou qualquer outra coisa. Segundo Arcand (1989), a sociedade Cuiva trata os velhos como se a velhice não existisse, evidenciando um modelo radicalmente oposto ao modelo ocidental, em que todos os meios são utilizados para acentuar a distinção entre as diferentes fases da vida.

As reflexões de Ellen Corin $(1982,1985)$ e de Gognalons-Caillard (1979) nos trazem de volta ao Ocidente. Segundo Corin (1985), a velhice é, geralmente, descrita no Ocidente em termos negativos (perda, falta) do que é valorizado socialmente. Gognalons-Caillard (1979) sugere que esta exacerbação da visão deficitária do envelhecimento estaria ligada à maneira pela qual a velhice no Ocidente situa-se na contracorrente de uma sociedade centrada na produção, no rendimento, na juventude e no dinamismo. Segundo Corin $(1982,1985)$, é a partir da idéia de perda (perdas de papéis sociais, perda de capacidades intelectuais) que se estruturaram os programas destinados aos idosos. A autora $(1982,1985)$ aler- 
ta também para a marginalização que uma definição tão negativa da velhice impõe aos idosos. Por esta razão, ela enfatiza que os estudos sobre o envelhecimento devem focalizar a articulação entre os parâmetros externos (ligados ao contexto sociocultural) e os parâmetros internos (ligados à vivência pessoal), bem como sua contribuição para construir uma relação individual com o envelhecimento.

Finalmente, devemos nos referir à contribuição da antropologia para o estudo dos fatores culturais que intervêm no campo da saúde. Embora sua contribuição situe-se em um nível bem mais geral, a antropologia médica fornece alguns elementos-chave para inovar a abordagem da saúde do idoso (Uchoa, 1997; Uchoa \& Vidal, 1994).

Nas últimas décadas, pesquisadores americanos e canadenses desenvolveram um importante quadro teórico e metodológico para estudar de maneira sistemática percepções e comportamentos predominantes neste campo. A introdução da perspectiva interpretativa no estudo destas questões constitui a base do corpo de conhecimentos da antropologia médica norte americana (Corin et al., 1992; Good, 1977; Kleinman, 1981). Um elemento central nesta abordagem é a distinção analítica entre "doença processo" (disease) e "doença experiência" (illness) (Kleinman, 1981). Esta distinção nos remete aos dois aspectos indissociáveis dos fenômenos saúde e doença, ou seja, o processo patológico e a experiência psicossocial deste processo, à importância de considerar que um aspecto não é redutível ao outro e às múltiplas e constantes interações que existem entre eles. Nesta abordagem, considera-se que a experiência da doença é uma construção cultural que conjuga normas de conduta, valores e expectativas tanto individuais quanto coletivas e se expressa em formas específicas de pensar e agir. Trata-se de investigar lógicas culturais subjacentes à identificação de problemas e às tentativas de resolução destes problemas, assim como dos diversos elementos do contexto (pessoal, social, e econômico) que podem influenciar a tradução destas lógicas em comportamentos concretos (Good \& Good, 1982; Kleinamn, 1981).

Um estudo desenvolvido recentemente entre idosos residentes na cidade de Bambuí, em Minas Gerais, ilustra a pertinência de integrar as contribuições da antropologia na investigação das questões relativas à da saúde do idoso (Uchôa et al., 2002). Neste estudo, utilizou-se uma abordagem interpretativa do envelhecimento e as informações foram obtidas através de entrevistas com informantes-chave e reconstruções de histórias de vida. Verificou-se que a avaliação da gravidade e da relevância de um problema de saúde pareciam ser claramente determinados pela possibilidade de enfrentá-lo, muito mais que pelo problema em si. Esta possibilidade estava associada ao apoio familiar e ao acesso a cuidados médicos. Para um grupo de senhoras de alto poder aquisitivo, a saúde não constituía um problema, apesar dos vários problemas de saúde por elas relatados (diabetes, doença de Chagas, fraturas, problema cardíaco). Com exceção destas senhoras, que podiam consultar médicos particulares, todas as outras relataram dificuldades para conseguir consultas no sistema público de saúde. A primeira dificuldade referia-se à existência de grandes filas para a retirada das fichas que garantem o acesso às consultas. Diante da impossibilidade de permanecer horas em pé nestas filas, várias senhoras relataram a necessidade de pagar a alguém para retirar as fichas para elas. O pagamento de consulta particular de um quarto, de um terço ou metade do preço regular - era outra estratégia desenvolvida, muitas vezes com grande esforço, por estas senhoras para evitar as grandes filas. Quando a questão da consulta era resolvida, surgia a do medicamento; poder comprar os remédios prescritos pelo médico era também visto como um fator essencial para o alcance ou manutenção da saúde. Assim, a situação econômica do próprio idoso e de sua família surgia como fator fundamental para a manutenção da saúde.

Esta representação da saúde como "dinheiro-dependente" reflete as desigualdades que caracterizam o acesso do brasileiro idoso aos serviços de saúde e aos medicamentos (LimaCosta et al., 2003). Evoca e, ao mesmo tempo, determina o desenvolvimento de estratégias resolutivas que podem ter repercussões negativas sobre a saúde do idoso, como a aquisição parcial do medicamento ou a redução da dose prescrita. Cabe aqui uma interrogação sobre o papel de uma representação deste tipo no contexto atual de promoção da saúde. Uma representação da saúde com sendo "dinheiro dependente" dificilmente incentivaria a população idosa a desenvolver hábitos mais saudáveis. Estas são certamente informações da maior importância para o planejamento de intervenções adequadas às características dessa população.

$\mathrm{O}$ estudo mencionado vem reafirmar a importância de compreender como o idoso vivendo em determinado contexto percebe sua saúde, o que ele define como problema e que estratégias usa para resolvê-lo. Nele, reafirma-se também a importância de contextualizar suas reações frente às perdas físicas (auditivas, visuais ou motoras), afetivas ou mudanças sociais e econômicas que caracterizam o avançar da 
idade. Finalmente, expressa-se, naquele trabalho, a necessidade de identificar os recursos (individuais e coletivos) disponíveis para que os idosos possam enfrentar estas dificuldades.

Uma abordagem antropológica das questões relativas à saúde de populações idosas nos obriga a ampliar nosso campo de estudo para incluir uma apreensão da experiência subjetiva e de sua interação com diversos elementos do contexto social e cultural. Trata-se, portanto, de uma ferramenta fundamental para a Saúde Pública contemporânea.

\section{Referências}

ARCAND, B., 1989. La construction culturelle de la vieillesse. In: Vieillir à Travers le Monde (R. Santerre \& G. Létourneau, ed.), pp. 97-106, SainteFoy: Les Presses de L'Université Laval.

BERQUÓ, E., 1999. Considerações sobre o envelhecimento da população no Brasil. In: Velhice e Sociedade (A. Liberalesso \& G. Debert, org.), pp. 11-40, Campinas: Papirus Editora.

BIRREN, J. E., 1983. Aging in America: Roles for psychology. American Anthropologist, 12:298-299.

CORIN, E., 1982. Régards anthropologiques sur la veillesse. Anthropologie et Sociétés, 6:63-90.

CORIN, E., 1985. Définisseurs culturels et repères individuels: Le rapport au corps chez les personnes agées. International Journal of Psychology, 20:471500.

CORIN, E.; UCHÔA, E.; BIBEAU, G.; KOUMARE, B.; COULIBALY, B.; COULIBALY, M.; MOUNKORO, M. \& SISSOKO, M., 1992. La place de la culture dans la psychiatrie africaine d'aujourd'hui. Paramètres pour un cadre de références. Psychopathologie Africaine, 24:149-181.

COWGILL, D. \& HOLMES, L. (ed.), 1972. Aging and Modernization. New York: Appleton Century Crofts.

DEBERT, G. G., 1999. A Reinvenção da Velhice. São Paulo: EDUSP.

DUCHARME, F., 1992. Impact du Soutien Conjugal et des Stratégies Adaptatives sur le Bien-être des Conjoints Agés. Rapport de Recherche. Montréal: Université de Montréal.

EVANS-PRITCHARD, E., 1989. Les systèmes de classe d'âge chez les Nuer. In: Vieillir à Travers le Monde (R. Santerre \& G. Letourneau, ed.), pp. 125-131, Sainte-Foy: Les Presses de L'Université Laval.

FOURNIER, J., 1989. Vieillir aux Etats Unis. In: Vieillir à Travers le Monde (R. Santerre \& Létourneau, ed.), pp. 139-148, Sainte-Foy: Les Presses de L'Université Laval.

GEERTZ, C., 1973. The Interpretation of Cultures. New York: Basic Books Inc.

GOOD, B., 1977. The heart of what's the matter: The semantics of illness in Iran. Culture, Medicine and Psychiatry, 1:25-58.

GOOD, B. \& GOOD, M. J. D., 1982. Toward a meaningcentered analysis so popular illness categories: "Fright-Illness" and "Heart Distress" in Iran. In: Cultural Conceptions of Mental Health and Therapy (A. J. Marsella \& G. White, ed.), pp. 141-166, Dordrechet: D. Reidel Publishing Co.

GOGNALONS-CAILLARD, M., 1979. La production sociale de la maladie dans la vieillesse. Gérontologie, 29:21-25.

GUEMPLE, L., 1989. La viellesse chez les inuit. In: Vieillir à Travers le Monde (R. Santerre \& G. Le- tourneau, ed.), pp. 107-112, Sainte-Foy: Les Presses de L' Université Laval.

KLEINMAN, A., 1981. Patients and Healers in the Context of Cultures. An Exploration of Boderland between Anthropology, Medicine and Psychiatry. Berkeley/Los Angeles: University of California Press.

LETOURNEAU, G., 1989. L'étude comparative du vieillissiment. In: Vieillir à Travers le Monde ( $\mathrm{R}$. Santerre \& G. Letourneau, ed.), pp. 13-30, SanteFoy: Les Presses de L'Université Laval.

LIMA-COSTA, M. F. F.; BARRETO, S. M.; GIATTI, L. \& UCHÔA, E., 2003. Desigualdade social e saúde entre idosos brasileiros: Um estudo baseado na Pesquisa Nacional por Amostra de Domicílios. Cadernos de Saúde Pública, 19:745-756.

MARSHALL, V., 1986. A sociological perspective on aging and dying. In: Later Life. The Social Psychology of Aging (V. Marshall, ed.), pp. 125-146, Beverly Hills: Sage Publications.

MARSHALL, V., 1987. Social perspectives on aging: Theoretical notes. In: Aging in Canada: Social Perspectives (V. Marshall, ed.), pp. 39-59, Markham: Fitzhenry \& Whiteside.

MEYERHOFF, B. \& SIMIC, A. (ed.), 1978. Life's Career Aging. Cultural Variations on Growing Old. Beverly Hills/London: Sage Publications.

NEUGARTEN, B. L., 1967. The aged in American society. In: Social Problems: A Modern Approach (H. S. Becker, ed.), pp. 179-189, New York: John Wisley and Sons.

UCHÔA, E., 1988. Les Femmes de Bamako (Mali) et la Santé Mentale. Une Étude Anthropopsyquiatrique. Thèse Ph.D. Montréal: Université de Montréal.

UCHÔA, E., 1997. Antropologia e epidemiologia. Contribuições para uma abordagem dos aspectos transculturais da depressão, In: Ciências Sociais e Saúde (A. Canesqui, org.), pp. 87-112, São Paulo: Editora Hucitec/Rio de Janeiro: ABRASCO.

UCHÔA, E.; FIRMO, J. O. A. \& LIMA-COSTA, M. F. F., 2002. Envelhecimento e saúde: Experiência e construção cultural. In: Antropologia, Saúde e Envelhecimento (M. C. S. Minayo \& C. E. A. Coimbra Jr., org.), pp. 25-35, Rio de Janeiro: Editora Fiocruz.

UCHÔA, E. \& VIDAL, J. M., 1994. Antropologia médica: Elementos conceituais e metodológicos para uma abordagem da saúde e da doença. Cadernos de Saúde Pública, 10:497-504.

VERAS, R. P., 1992. A Survey of the Health of Elderly People in Rio de Janeiro, Brazil. Ph.D. Thesis. London: University of London.

Recebido em 29 de abril de 2002

Versão final reapresentada em 12 de agosto de 2002

Aprovado em 10 de outubro de 2002 